\title{
A Practical Approach for Implementing Business Process Reengineering
}

\author{
Dr. Ahmed El-abbassy \\ El-shorouk Academy, Institute of Computers and Information Technology \\ P.O Box, El-shorouk, Cairo, Egypt \\ ahmed_elabbassy@yahoo.com
}

\begin{abstract}
:
Business Process Reengineering (BPR) projects with objectives to drastically restructure business processes are not successful. Sources of failure are multiple.

An important source of failure is that, BPR focuses on processes and ignores other important aspects of institutions such as organizational structure, resistance to change, people, communication, and technology.

Many organizations take the decision to delay and/or abandon BPR projects due to: - Implementing BPR means being involved in a drastic restructuring of organization structure and processes.

- A full scale reengineering effort involves substantial cultural change.

-Implementing BPR requires collecting data and measures which are not always accurately available for many organizations.

In this paper, we discuss a practical approach to implement BPR that takes into consideration those important institutional factors.

In this approach, we propose to place significant emphasis on workflow management systems as a major enabler to BPR.

The workflow management systems act as the engine around which BPR is implemented. Corporate measures could be collected from the actual implementation of the current processes, then analyzes and changes are progressively decided based on these actual measures. This will allow a smooth, flexible and effective change process.
\end{abstract}

Keywords: Business Process Reengineering, Workflow Management System.

\section{Business Process Reengineering (BPR) $[1,2.3]$}

\subsection{BPR Definition}

Business Process Reengineering (BPR) is defined as the fundamental rethinking and radical redesign of business processes to achieve dramatic improvements in critical contemporary measures of performance such as cost, quality, service and speed.

BPR project attempts to redesign a hierarchical company of functional "silos" into a process-based organization in which activities are organized around a 
particular outcome and then implementing the resulting changes in company structures, activities, information systems and attitude.

\subsection{BPR Implementation Process}

As illustrated in figure (1), the steps to Reengineering are:

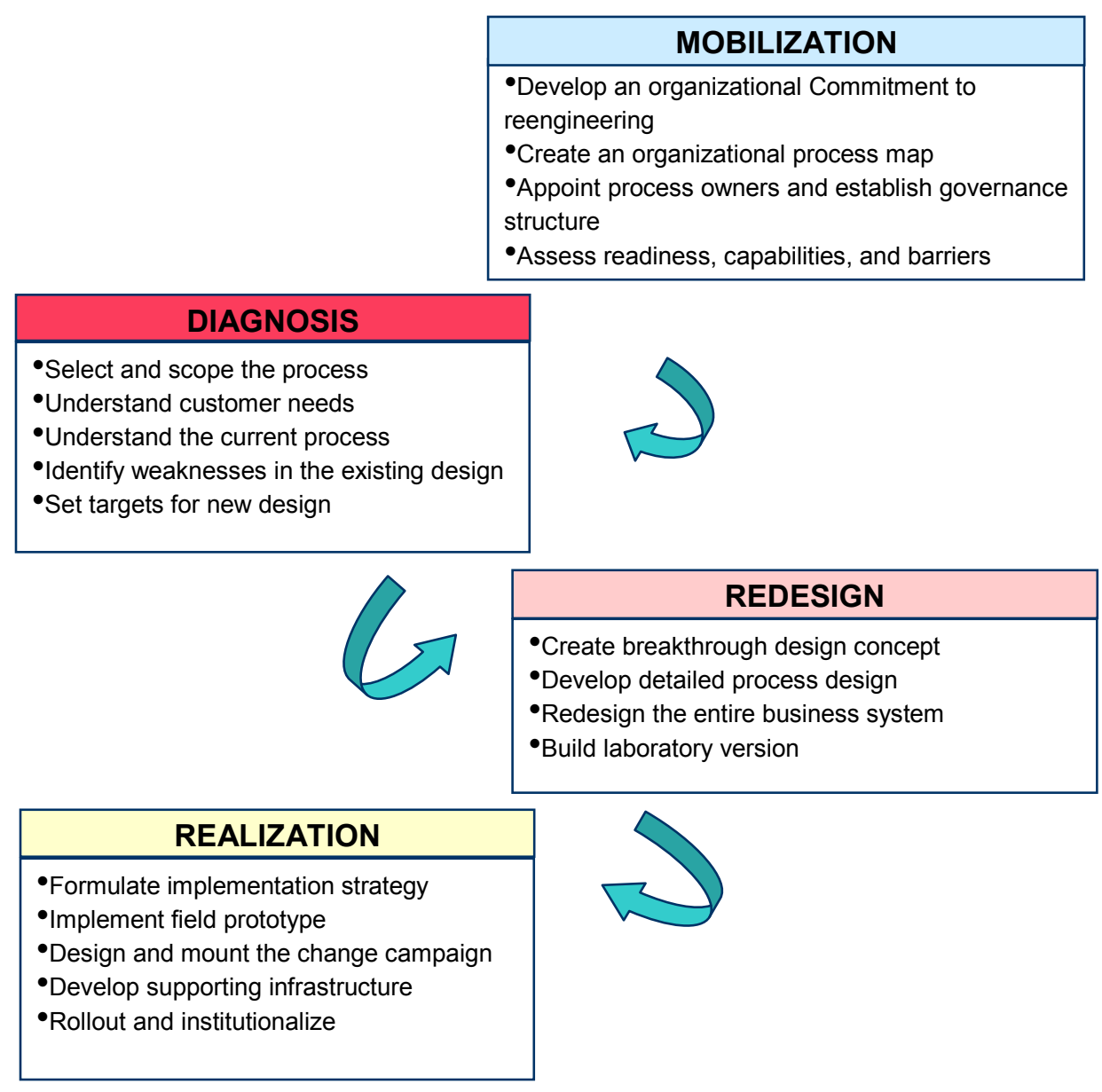

Figure (1) Reengineering Steps

\subsection{Redesign issues}

- Enforcing standards

- Changing from personal to workgroup computing

- Performance gains (Responsiveness)

- Re-casting external relationships (to focus upon the key areas of business, support the move to outsourcing)

- Development of new high performance work models

- Quality (Enhancing consistency and predictability) 
A Practical Approach for Implementing Business Process Reengineering

- Facilitate cross-sectional data flow and removal of sectional based systems.

- Aligning support services with essential business processes to lead to radical benefits

\subsection{BPR metrics}

Measures are:

- Speed (reduced cycle time)

- Reduction in paper handling

- Ease of access to service

- Reduced cost

\section{Workflow Management Systems $[4,5,6]$}

\subsection{Workflow Definition}

Workflow management systems automate the capturing, routing, processing and management of all objects (data, documents, electronic forms, text, voice, etc,).

The workflow software makes automation of workflow possible. The automation of workflow can be implemented at several levels. At the basic level, workflow software performs the routing function similar to an electronic mail system. The objects are forwarded to various users for processing and the last user completes the work and closes the task sequence. However, the complexity of business operations require more sophisticated workflow software with advanced work management functions

\subsection{Workflow Components}

The Workflow management system may encompass many functions and components- However a typical workflow management system has the following components as illustrated in figure (2):

- Routing

- Queuing

- Retrieval

- Processing

- Administrative Controls

- Development Utilities 


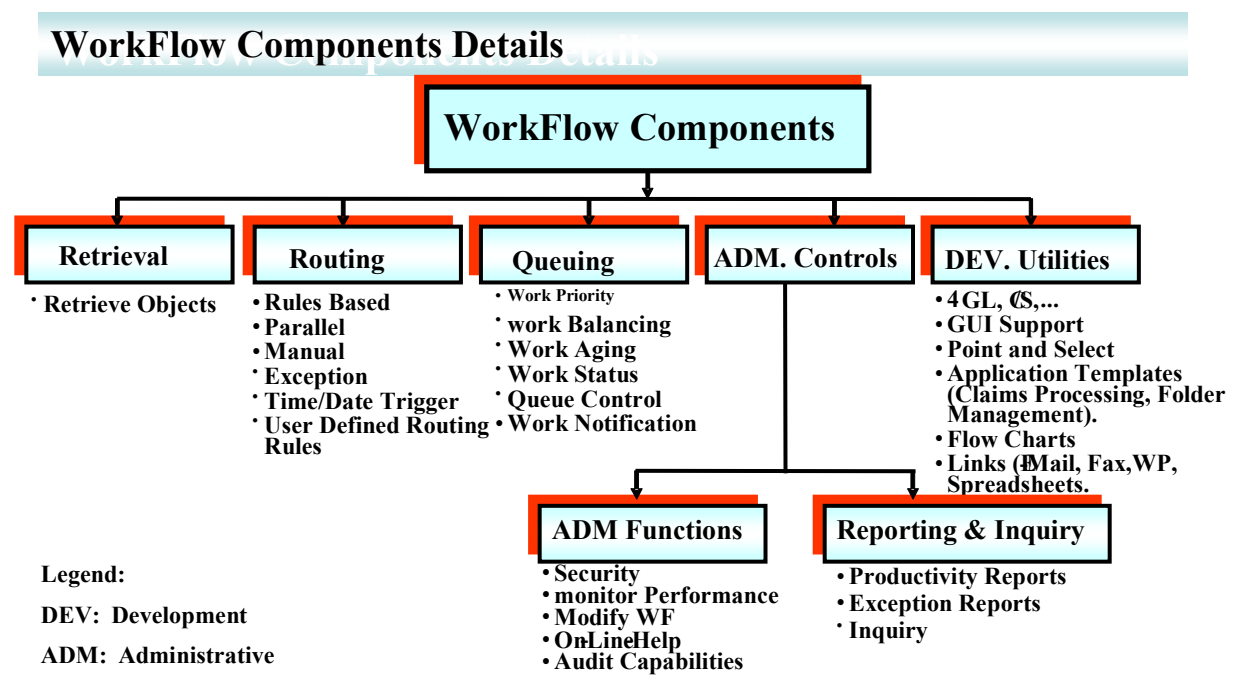

Figure (2) Components of Workflow management system

\subsection{Workflow management meta-model}

Workflow Management Systems are considered as strategic technologies to improve business processes, increase productivity, reduce cost and improve the product or service supplied.

The meta-model of workflow management consists of a process model and an organization model. The organization model is composed of organizational units, roles and individuals. These entities are assigned as process performers to entities of the process model. The process model is composed of processes, process activities (sub process), block activities (loop) and program activities (application).

A process type is defined in the build-time environment. A process instance is executed in the runtime environment according to its process definition. Workflow management systems can be seen as state event machines; process and activity instances get through events into certain predefined states. All events in the life cycle of a process instance or an activity instance are recorded in an audit trail. Each record in the audit trail contains a number of information including, but not limited to following items: date and time when the event takes place, type of event, process identifier, activity type, activity state, activity identifier, started program name, role or id associated with the event, etc.

\subsection{Integrating BPR with flow management systems}

Almost every BPR engagement begins with a 'process capture' phase. 


\section{A Practical Approach for Implementing Business Process Reengineering}

Workflow management systems are used as analysis tool to assess current processes and to simulate new processes. It allows reengineering team to:

- Draw workflow maps.

- Analyze performance by cycling couriers through a virtual model based on the rules and routing information defined in workflow maps. Activities on the map are emulated.

- Display the status of work flowing through the activity maps on the system.

- Track the history of a courier through the system.

The use of Workflow management system greatly reduces the time involved in creating a simulation model suitable for identifying bottlenecks and idle resources in a complex workflow.

Ultimately, the implementation of the re-engineered processes may employ a workflow management system.

\section{Definition of the problem $[1,7,8]$}

Approximately $70 \%$ of BPR projects fail and some believe that this figure may be even higher. Sources of failure are multiple and are documented in the literature.

An important source of failure is that, BPR focuses on processes and ignores other important aspects of institutions such as organizational structure, resistance to change, people, communication, and technology.

Many organizations take the decision to delay and/or abandon BPR projects due to:

- Implementing BPR means being involved in a drastic restructuring of organization structure and processes.

- A full scale reengineering effort involves substantial cultural change.

- Implementing BPR means collecting a lot of data and measures which are not always available for many organizations.

As a conclusion, BPR fails due to the ignorance of the human side of organizations.

Reengineering treated the people inside companies like bits and bytes, interchangeable parts to be reengineered.

Because people are fundamental to most organizational processes, social and psychological issues must be considered, as must the existing resources and interpersonal relationships in the organization.

There is a need to find out practical ways to implement BPR that take into consideration those important institutional factors.

We believe that workflow management systems can play a proactive role for a smooth, gradual and successful implementation of BPR.

Though there is general agreement that the automation of workflow is a key ingredient of any process reengineering effort, there is no clear way how to use workflow management systems to implement a successful BPR. 


\section{Use of workflow management systems to enable BPR $[9,10,11,12]$}

Workflow management systems are emerging infrastructure technology that can facilitate the BPR initiative. Workflow management systems can be seen as strategic technology to change culture. We propose to place significant emphasis on workflow management systems as a major enabler to BPR.

The workflow management system tracks any event change with a timestamp in the workflow history log or audit trail. As a consequence, workflow management systems facilitate workflow monitoring and controlling and play a key role in measuring the efficiency of business processes, which is crucial for the success of continuous improvement. The audit trail of the workflow management system is stored in a database table and can be accessed easily.

We consider the workflow management systems the engine around which BPR is implemented. Corporate measures could be collected from the actual implementation of the current processes, then analyzed and changes are progressively decided based on these actual measures. This will allow a smooth, flexible and effective change process.

This paper proposes a practical approach to implement Workflow management systems.

The change process is illustrated in figures ( 3 and 4 ).

\section{The Change Process}

\section{Start}

1. Set Performance Figures for Each Workflow Step

2. Implement Current Workflow Cycles

\section{Repeat}

3. Collect Data Concerning Actual Workflow Steps [Benchmarking]

4. Study the Actual Performance Figures

If Actual Figures are Satisfactory

Then

\section{End}

Else

5. Revisit the Workflow Cycles for improvement

6. Implement New Workflow Cycles

Figure (3) Workflow management -enabled BPR 
A Practical Approach for Implementing Business Process Reengineering

The change process encompasses the following steps:

\subsection{Set Initial Performance Figures for Each Workflow Step}

A hard obstacle when we start any BPR initiative is to collect data about the time of performing each workflow step.

With workflow enabled BPR, this is flexible, we can start with initial performance figures estimated by the managers responsible for the different working procedures, or even we can start without setting performance figures, and let these figures be collected from the actual implementation of the workflow system.

\subsection{Implement Current Workflow Cycles}

With workflow management- enabled BPR, redesign of business processes is not a precondition to start the actual use of workflow system. We can start by implementing the current workflow procedures and or introduce slight modifications for some cycles with apparent symptoms.

The improvement of workflow cycles is an inherent activity within the workflow management-enabled BPR process.

\subsection{Collect Data Concerning Actual Workflow Steps [Benchmarking]}

Measurements are the key to implement any change. Workflow management systems allow collecting a comprehensive and timely set of performance figures that can be used to discover the cycles that need improvement.

Corporate performance measurements focused on business processes are inherent capability in the workflow management systems.

The performance-relevant data collected are stored on a non-volatile media (e.g. a relational database) so that the data can be analyzed over a long period of time. Performance data stored in a workflow management system can be accessed by different levels of staff such as process owners or general managers.

A lot of business questions can be answered with audit trail data tracked by the Workflow Management System.

\subsection{Study the Actual Performance Figures}

The actual performance figures are analyzed in order to check the need for change.

It is possible to integrate with additional approaches such as a data warehouse approach applied to workflow history data.

\subsection{Revisit the Workflow}

If the current performance figures are unsatisfactory and a need for change is emerged, the workflow cycle is redesigned. 


\subsection{Implement New Workflow Cycles}

The new workflow cycle is implemented

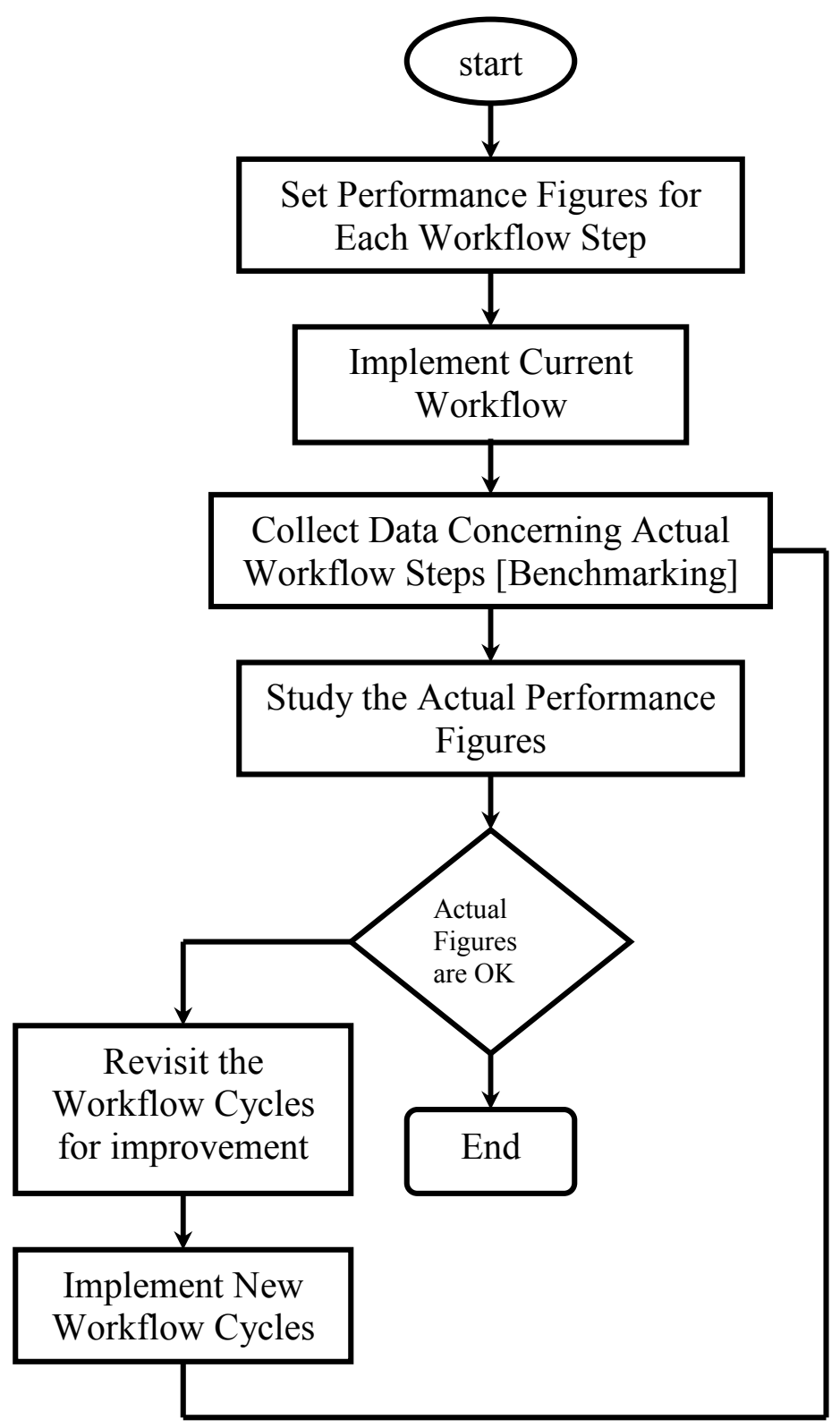

Figure (4) Workflow management-enabled BPR process 
A Practical Approach for Implementing Business Process Reengineering

\section{Conclusion}

This paper illustrated a practical approach to implement incrementally BPR based on the use of workflow management systems. By this way workflow management systems are implemented without waiting for a lengthy BPR as a precondition. Workflow management systems can help in preparing the organizations for change. Through this approach the organization performance figures could be collected during the operations of the Workflow management system and changes are introduced based on the collected figures. This will allow smooth, flexible and effective implementations of Workflow management systems and BPR projects.

We expect to extend this approach to define more detailed relationships between the BPR redesign issues with the figures collected from the workflow management systems.

\section{References}

[1] Delvin Grant, A wider view of business process reengineering, Communications of the ACM, Volume 45, Number 2 (2002), Pages 85-90

[2] Hammer, M. and Champy, J. Reengineering the Corporation. Harper Collins, NY, 1993.

[3] Ritu Agarwal and Michael Albers, The Practice of Business Process Reengineering: Radical Planning and Incremental Implementation in an M Organization, SIGCPR-3/94- Alexandria, Virginia, USA,1994 ACM 0-89791- 852-2/94/0003.

[4] Paul Dourish, Process Descriptions as Organizational Accounting Devices: The Dual Use of Workflow Technologies, GROUP'01, Sept. 30-Oct. 3, 2001, Boulder, Colorado, USA., Copyright 2001 ACM 1-58113-294-8/01/0009, Pages52-60.

[5] Robert M. Shapiro, Integrating BPR with image-based workflow, Proceedings of the 1994 Winter Simulation Conference, Pages 1222-1228.

[6] Keith D Swenson, Workflow Technology: Tradeoffs for Business Process Reengineering, COOCS 95 Milpitas CA USA (c) 1995 ACM 0-89791-706-5/95/08, Pages 22-29.

[7] James T.C. Teng, Seung Ryul Jeong, and Varun Grover Profiling Successful Reengineering Projects, June 1998/Vol. 41, No. 6 COMMUNICATIONS OF THE ACM, Pages 96-102.

[8] John Brier and Lucia Rapanotti, Problem-based Analysis of Organizational Change: a Real-world Example, IWAAPF'06, May 23, 2006, Shanghai, China. Copyright 2006 ACM 1-59593-085-X/06/0005, Pages 13-18.

[9] Gary Katzenstein and F. Javier Lerch, Beneath the Surface of Organizational Processes: A Social Representation Framework for Business Process Redesign, ACM Transactions on Information Systems, Vol. 18, No. 4, October 2000, Pages 383-422. 
Journal of the ACS , Vol. 1, June 2007

[10] Joachim Schelp, Alexander Schwinn, Extending the Business Engineering Framework for Application Integration Purposes, 2005 ACM Symposium on Applied Computing, Pages1333-1336.

[11] Beate List, Karl Machaczek, Towards a Corporate Performance Measurement System, 2004 ACM Symposium on Applied Computing, pages 1344-1350.

[12] Rajiv Kohli and Ellen Hoadley, Towards Developing a Framework for Measuring Organizational Impact of IT- Enabled BPR: Case Studies of Three Firms, The DATA BASE for Advances in Information Systems - Winter 2006 (Vol. 37, No. 1), Pages 40-58. 